\title{
Design and Development of Advanced Air-Cathodes for Li-Air Battery
}

\author{
Dr. James J. Wu \\ Photovoltaic and Electrochemical Systems Branch, Power Division \\ NASA Glenn Research Center
}

Presentation at AiMES 2018 Meeting Cancun, Mexico September 30 - October 4, 2018 


\section{Outline}

- Introduction/NASA Energy Requirements

- Challenges for Li-Air Battery \& Air-Cathode Development

- Design Parameters for Air-Cathode

- Result Summary

- Next Steps/Future Direction 


\section{Batteries: Important for NASA Missions}

- Batteries provide:

- a versatile, reliable, safe and portable energy source, and are an essential component of the power system of virtually all NASA missions

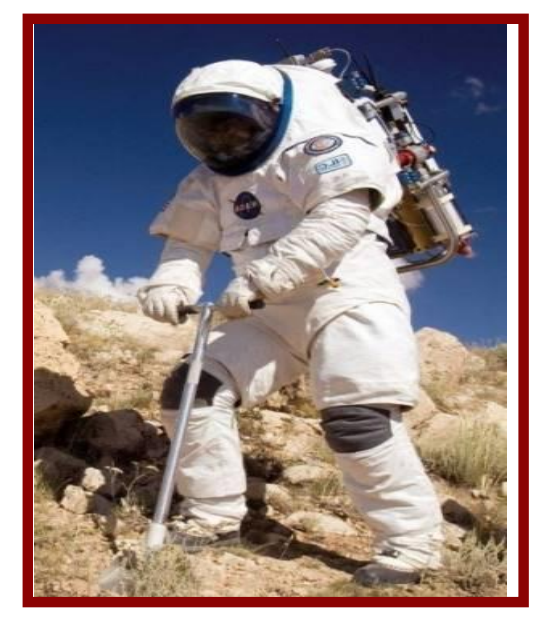

- energy storage, serve as a power source such as during eclipses, and can provide peaking power, which are important electrical energy storage options for NASA space missions

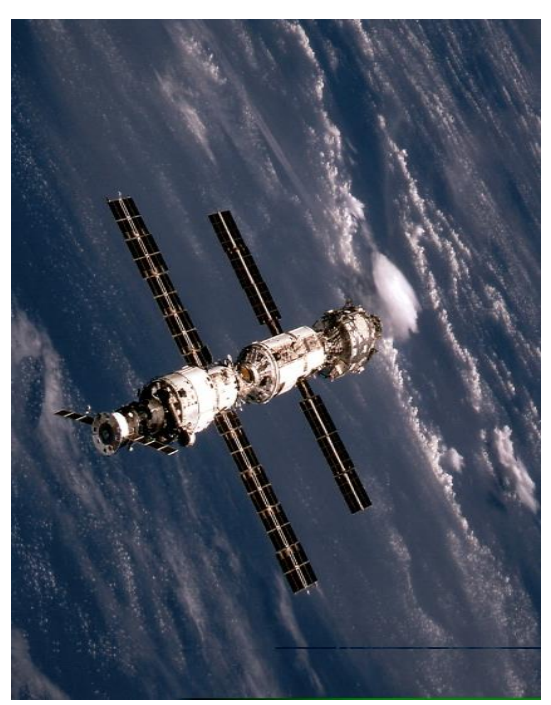




\section{Desired Battery Properties for NASA Missions}

- Safe

- High in specific energy

- Light in weight

- Compact in volume

- Long in shelf life

- Durable in wide temperature ranges and harsh environments

- Reliable in meeting mission requirements 


\section{State-of-Art (SOA) Li-Ion Battery (LIB)}

- LIB Specs:

- Specific energy: 180-200 Wh/kg

- Specific power: $300 \mathrm{~W} / \mathrm{kg}$

- Temp range: $-20^{\circ} \mathrm{C}$ to $60^{\circ} \mathrm{C}$

- Excellent rechargeability: (1000s cycles)

- Limitations:

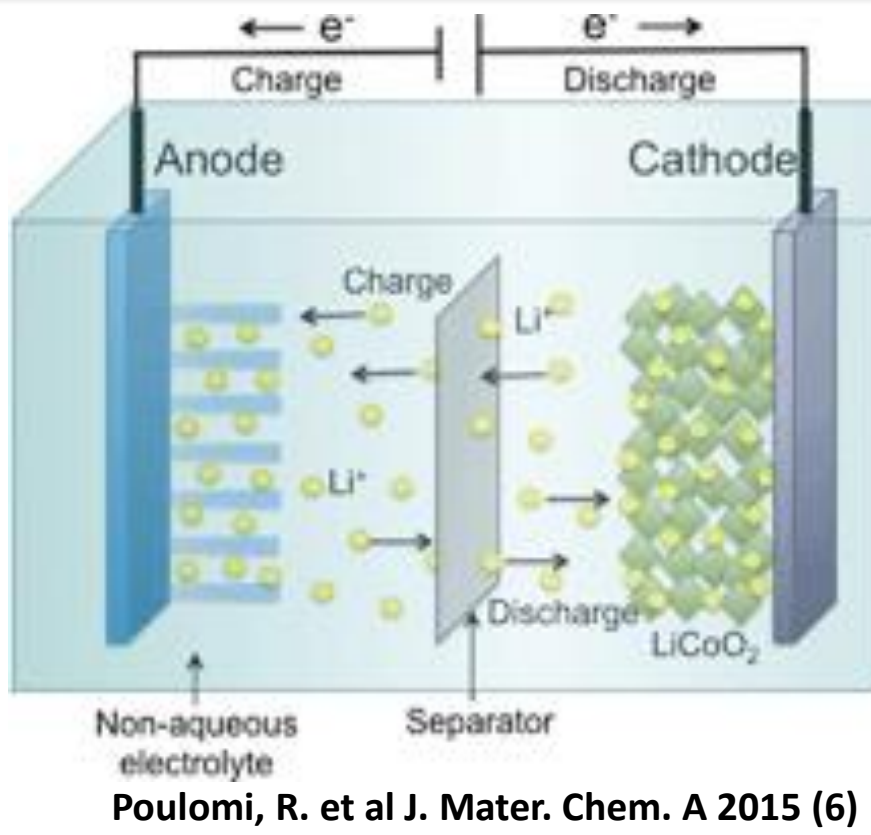

Anode: Graphite

Cathode: $\mathrm{LiCoO}_{2}$

Electrolyte: Li salt in organic carbonate

- Maximum of specific energy $<250 \mathrm{Wh} / \mathrm{kg}$

- Flammable electrolyte and fire hazards 


\section{NASA Demands Very High Specific Energy Batteries}

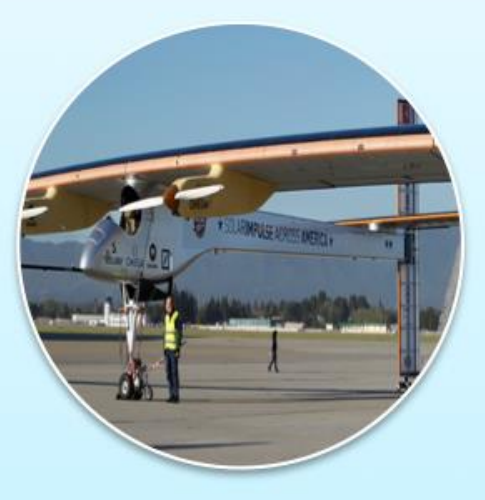

Electric Aviation $500-750 \mathrm{Wh} / \mathrm{kg}$

- Green aviation - Less noise, lower emissions, high efficiency

- Hybrid / All-electric aircraft Limited by mass of energy storage system

- Commercial aviation - Safe, reliable, lightweight on-board electric auxiliary power unit

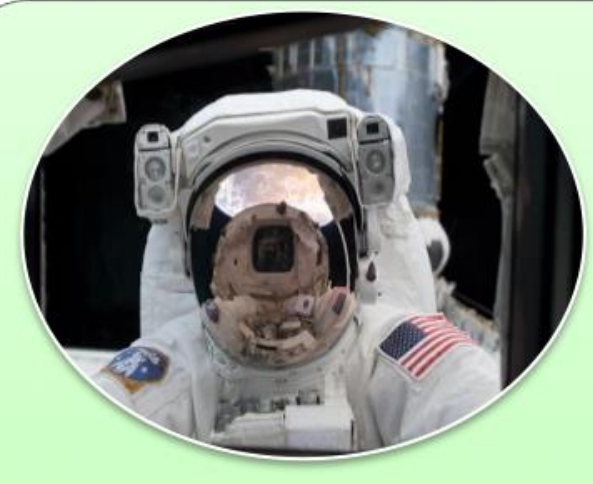

Extravehicular Activities

(Spacesuit power)

$$
>400 \mathrm{Wh} / \mathrm{kg}
$$

Required to enable untethered EVA missions lasting $\mathbf{8}$ hours within strict mass and volume limitations.

Astronaut life support

Safety and reliability are critical 100 cycles

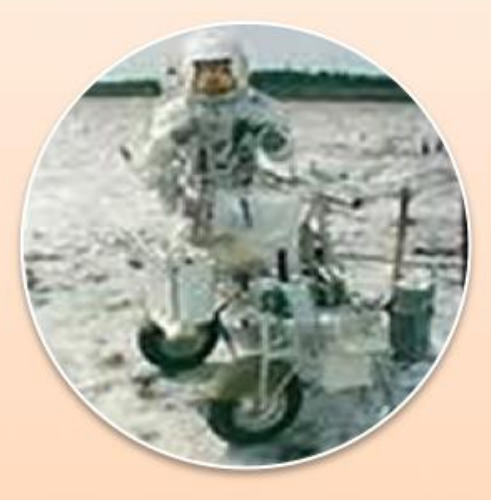

Landers and Rovers, Robotic missions, Inspace habitats

$$
>500 \mathrm{Wh} / \mathrm{kg}
$$

Batteries are expected to provide sufficient power for life support and communications systems, and tools including video and lighting

- >100 cycles

NASA future mission requirements far exceed the capabilities of SOA Li-ion chemistries

$>$ Progress in these areas requires advances in safe, very high energy batteries 


\section{Advanced Safe, High Energy Batteries}

- Improve Li-ion specific energy and safety

- Advanced electrode materials development such as:

- Si anode

- NMC cathode

- non-flammable additives in electrolyte

- under NASA Advanced Space Power System (ASPS) project (2009-2014) and under NASA Advanced Energy Storage Systems (AESS) project (2014-2017)

- Beyond Li-ion battery chemistries development

- NASA Center Innovative Fund (CIF)

- Independent Research and Development (IRAD)

- Convergent Aeronautics Solutions (CAS)

- NASA SBIR/STTR Program 


\section{Beyond Li-Ion Battery Chemistries}

- Battery chemistries with very high theoretical specific energy:

- Li/S Battery Chemistry: $2680 \mathrm{Wh} / \mathrm{kg}$

- Metal-Air Battery chemistries, e.g. Li/O $\mathrm{O}_{2:} 5200 \mathrm{Wh} / \mathrm{kg}$

- These high theoretical capacity battery chemistries have the potential to meet NASA's energy goal of $>400 \mathrm{Wh} / \mathrm{kg}$ 


\section{Li- $\mathrm{O}_{2}$ Battery Chemistry: Challenges}

- Complex discharge intermediates/ products: $\mathrm{LiO}_{2}, \mathrm{Li}_{2} \mathrm{O}_{2}, \mathrm{Li}_{2} \mathrm{O}$

- High overpotential during charge

causing electrolyte decomposition

- low cycle life

- Low kinetics: poor rate capability

- air cathode is "open" to environmental

- environment e.g. $\mathrm{CO}_{2}$, humidity, air flow, temperature impact the battery performance

- Safety, Li dendrite growth

- Air cathode and electrolyte development - critical for Li- $\mathrm{O}_{2}$ battery

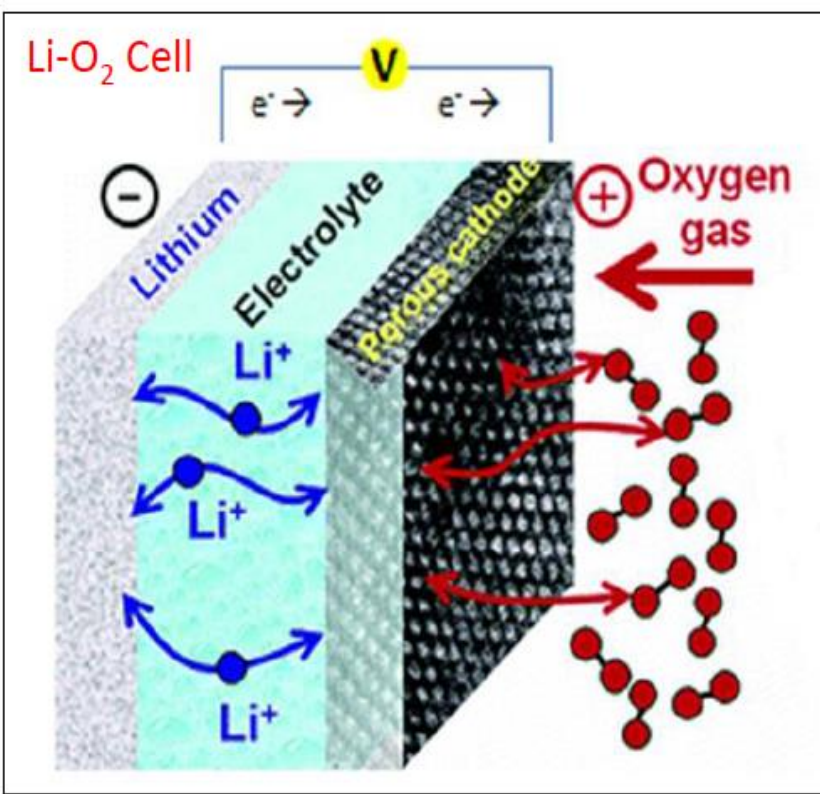




\section{Air-Cathode Design}

- Requirement for the air-cathode:

- Permeable to air $/ \mathrm{O}_{2}$ - porous substrate

- High catalytic reaction:

- oxygen reduction reaction (ORR)

- oxygen evaluation reaction (OER)

- Substrates

- porous with adequate mechanical strength

- good electronic conductivity

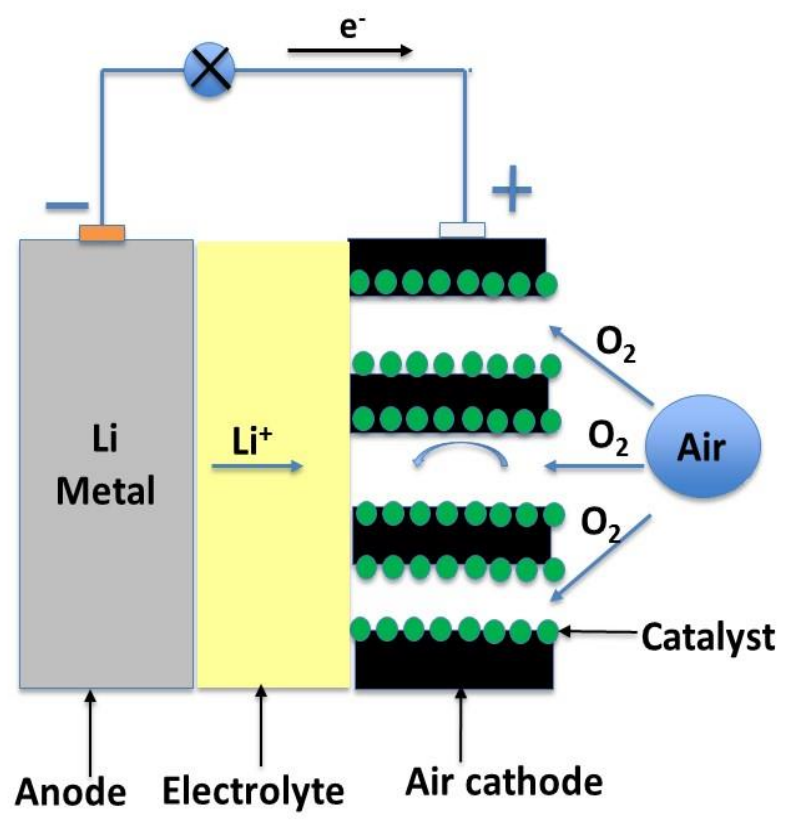

- stable chemically to electrolyte an discharge intermediate/products

- Stable at wider electrochemical window

- Plus if the substrate has catalytical activity to $\mathrm{O}_{2}$

- Active materials of cathode

- High surface, high porosity and high catalytical activity to $\mathrm{O}_{2}$

- Stable chemically and at wider electrochemical window 


\section{Air-Cathode Development}

- Different substrates

- 2D carbon: (carbon paper/cloth), 2D metal mesh (Ti, Pt/Ti)

- 3D graphene, 3D Ni foam. 3D Ni foam with CVD multilayer 3D graphene

- Different carbon as active materials

- Super P

- KetjinBlack (KB) carbon

- Catalyst in cathode

- Metal oxide

- Nobel metal

- Porosity of Ni Foam Substrate

- Different porosity

- Additive in electrolyte 


\section{Different Substrate Study}

\section{D Graphene/3D Ni foam}

\section{D Ti mesh}
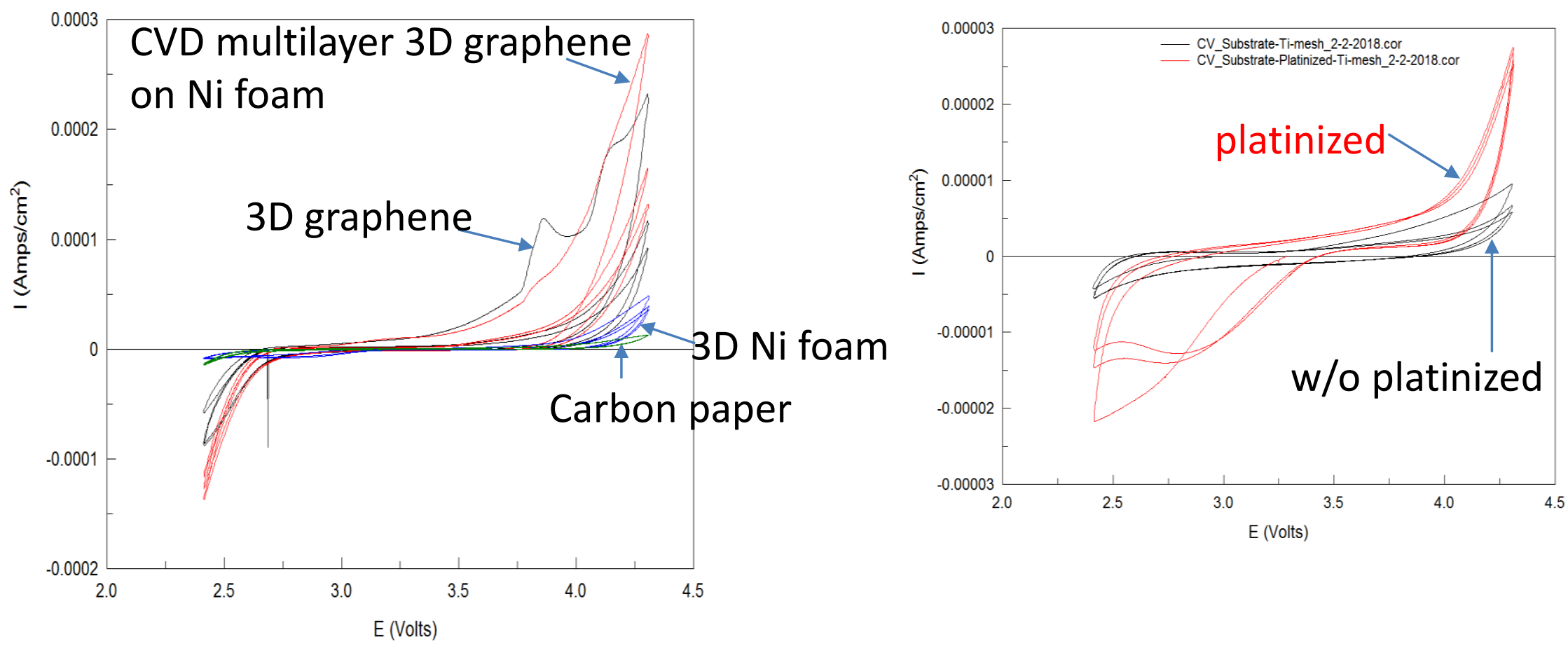
- S-P_cathode-2A_LiTf-TEGDME_CV-0.1mV-per-sec_11-7-2017.cor

- - S-S-P_cathode-1B_LiTf-TEGDME_CV-0.1mV-per-sec_11-8-2017.cor

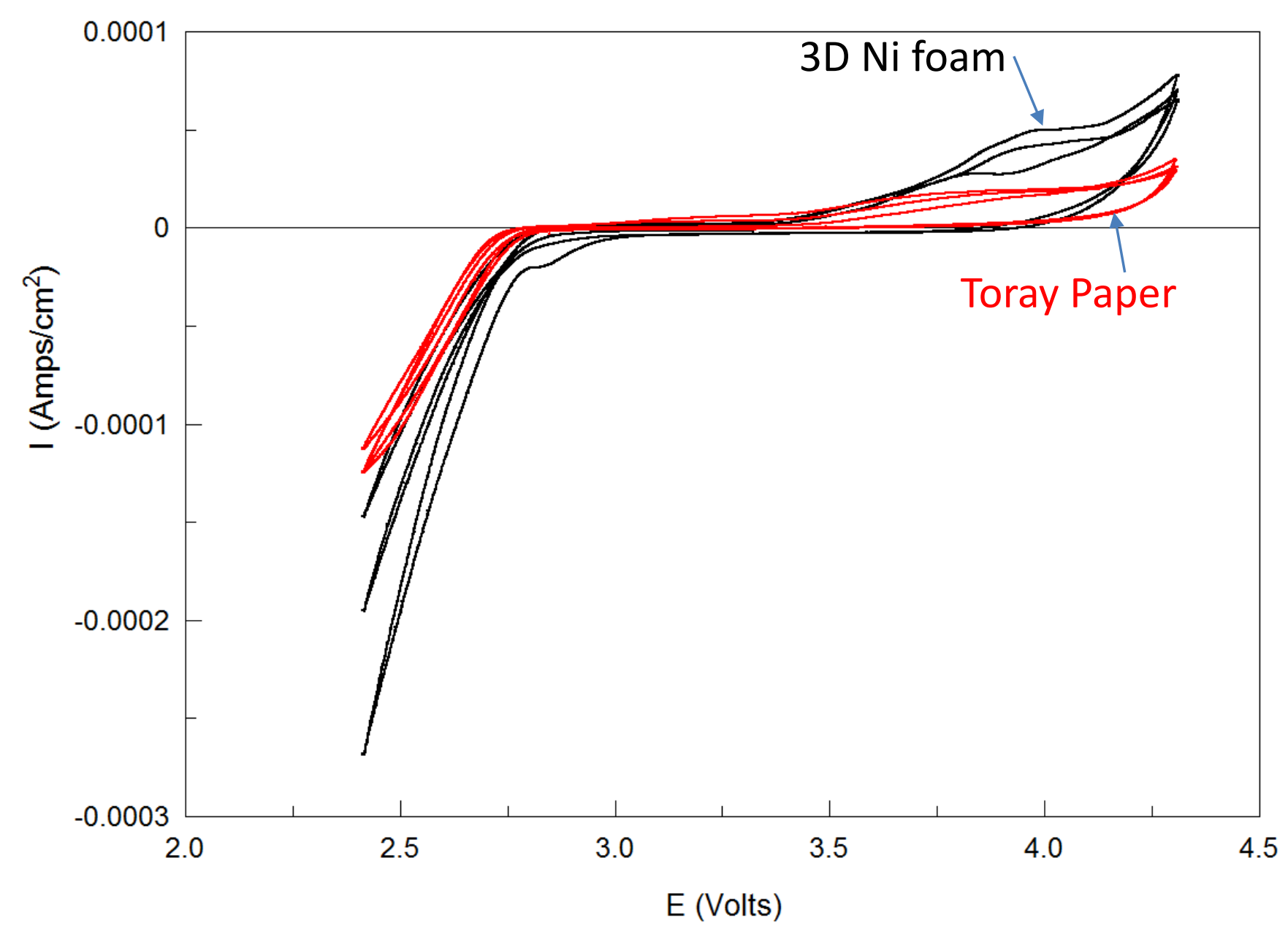




\section{Cyclic Voltammetry of Carbon (KB) on Ni Foam}

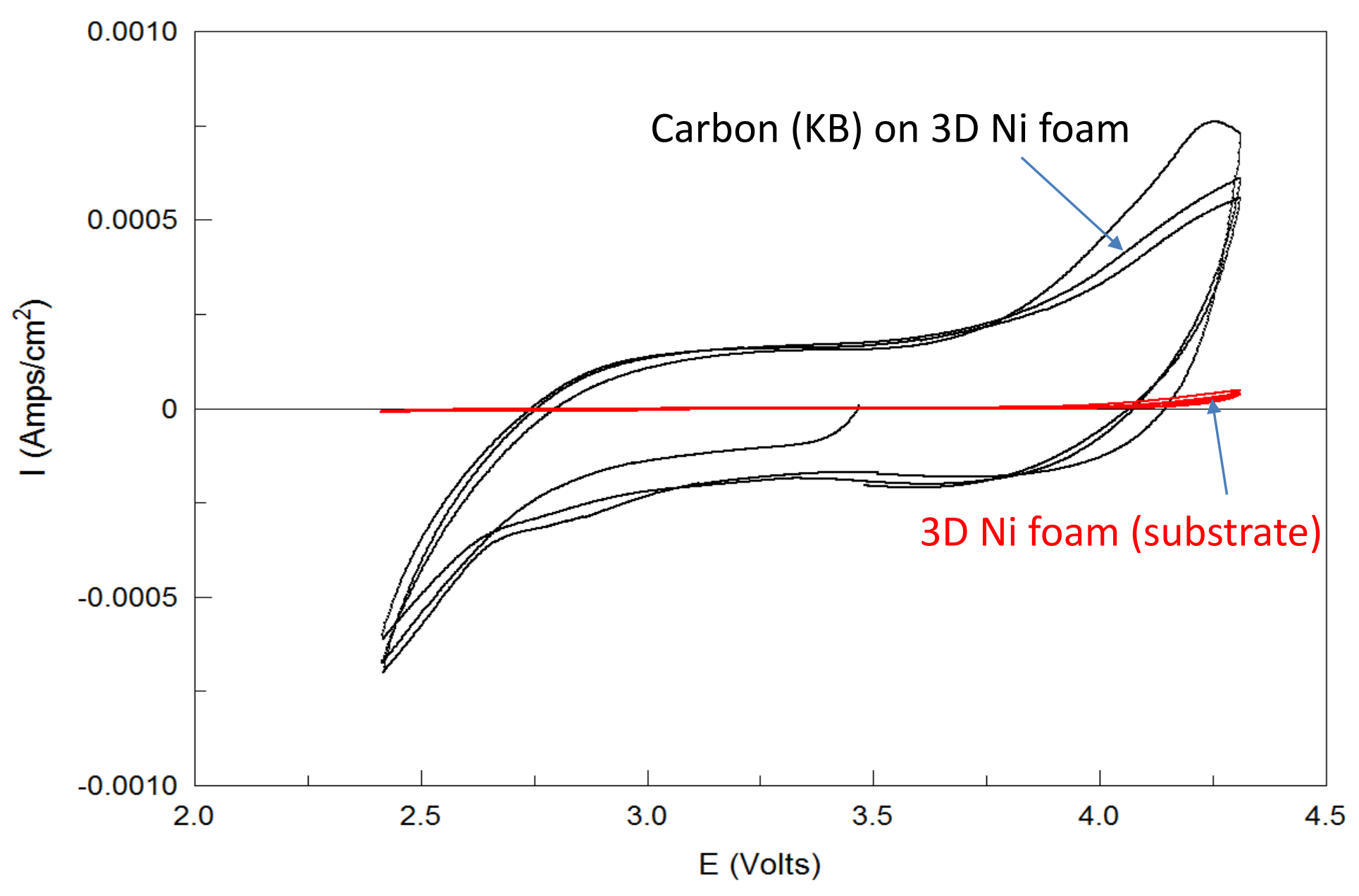




\section{Substrates and Active Materials Study}

- Platinized Ti mesh is better than Ti mesh w/o Pt treatment

- 3D Ni foam is better than 2D carbon/metal mesh

- Ketjin Black carbon is better than super P carbon

- 3D Ni foam and Ketjin Black are selected as cathode material 


\section{Porosity Impact}

\section{$75 \mathrm{~mA} / \mathrm{g}$}

Ni foam pore size: 450 um

Ni foam pore size: 680 um
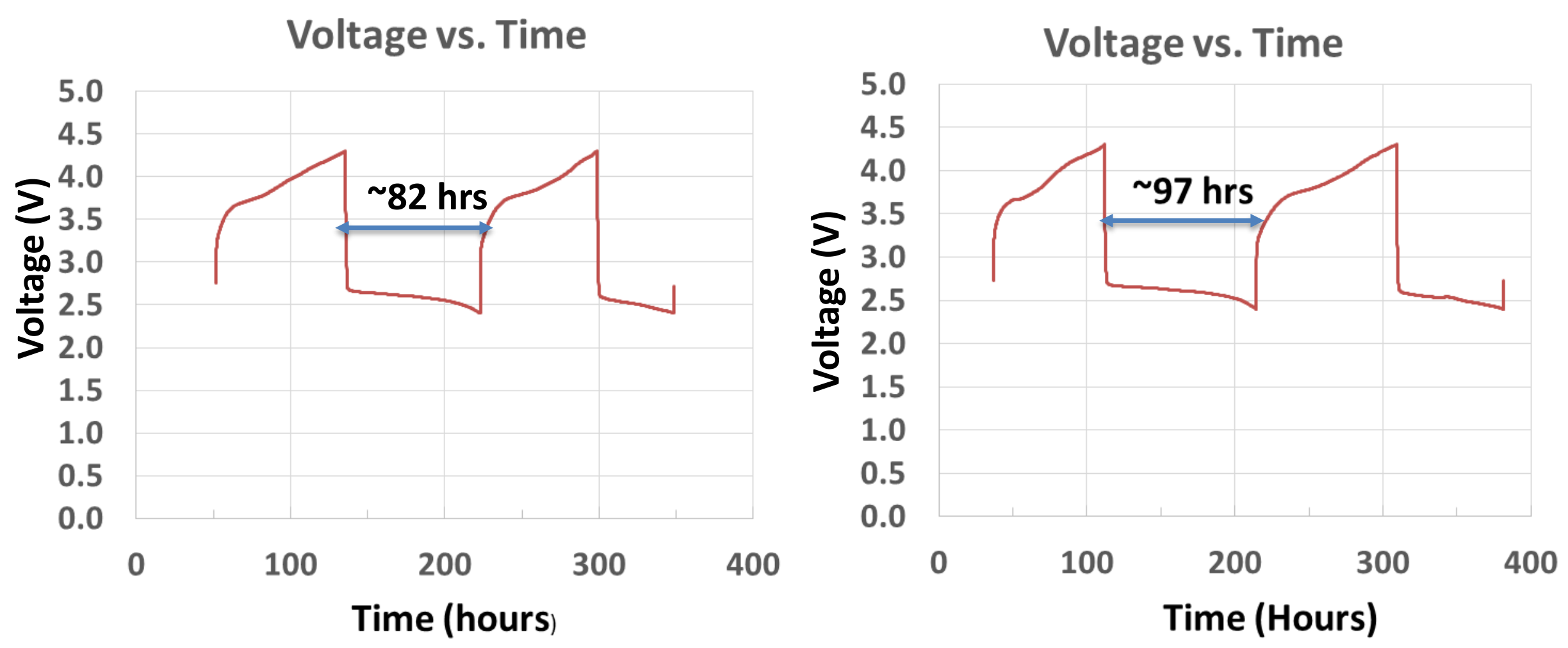


\section{(496) Cycling Performance at Different Cut-off Voltage}

\section{Current: 450 mA/g}
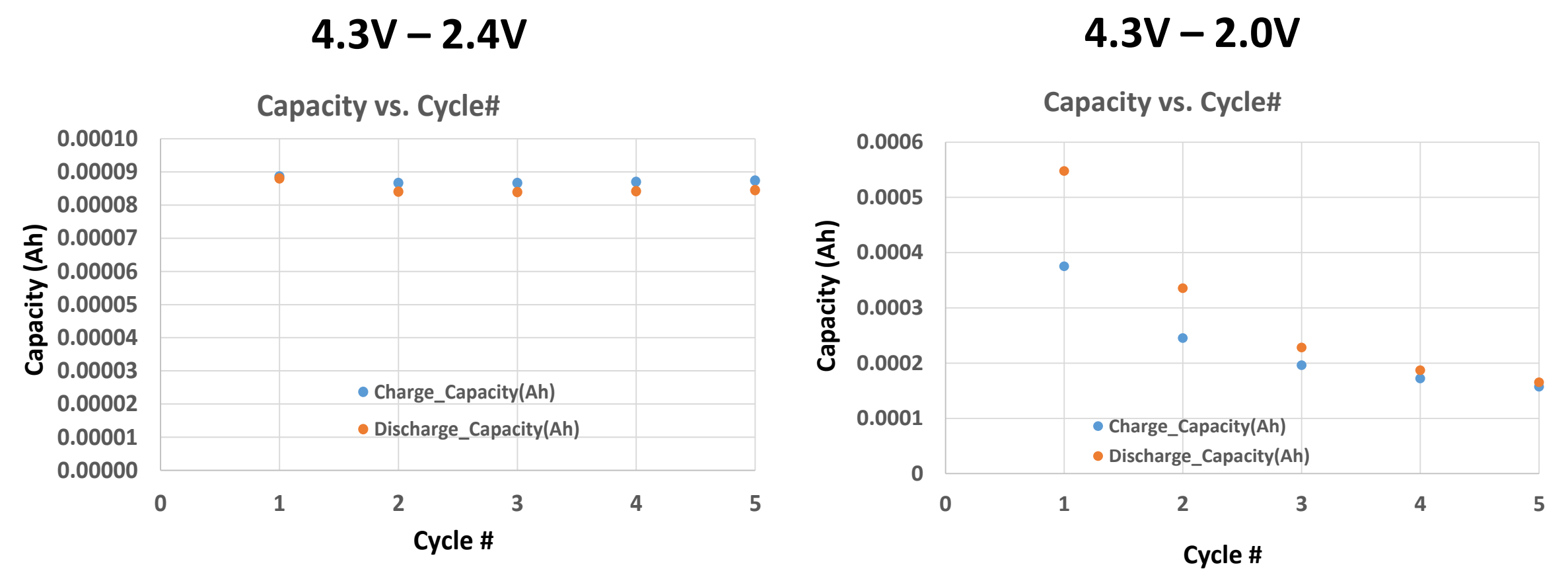


\section{Rate Capability Cycling of Li-Air Cell}

- KetjenBlack carbon on 3D Ni foam

- Loading: $3.2 \mathrm{mg} / \mathrm{cm}^{2}$

- Current density (mA/g):

$150 \rightarrow 300 \rightarrow 450 \rightarrow 300 \rightarrow 150$

$150 \mathrm{~mA} / \mathrm{g}---\rightarrow 0.47 \mathrm{~mA} / \mathrm{cm}^{2}$

$300 \mathrm{~mA} / \mathrm{g}--\rightarrow 0.95 \mathrm{~mA} / \mathrm{cm}^{2}$

$450 \mathrm{~mA} / \mathrm{g} \rightarrow 1.42 \mathrm{~mA} / \mathrm{cm}^{2}$

- $4.3 \mathrm{~V}-2.4 \mathrm{~V}$

- Discharge: $1 \mathrm{hr}$ or $2.4 \mathrm{~V}$

- High purity air (water <1 ppm in total contamination) environment 


\section{$150 \mathrm{~mA} / \mathrm{g}$ : Before and After Rate Capability Cycling}

\section{$150 \mathrm{~mA} / \mathrm{g}$, initial}

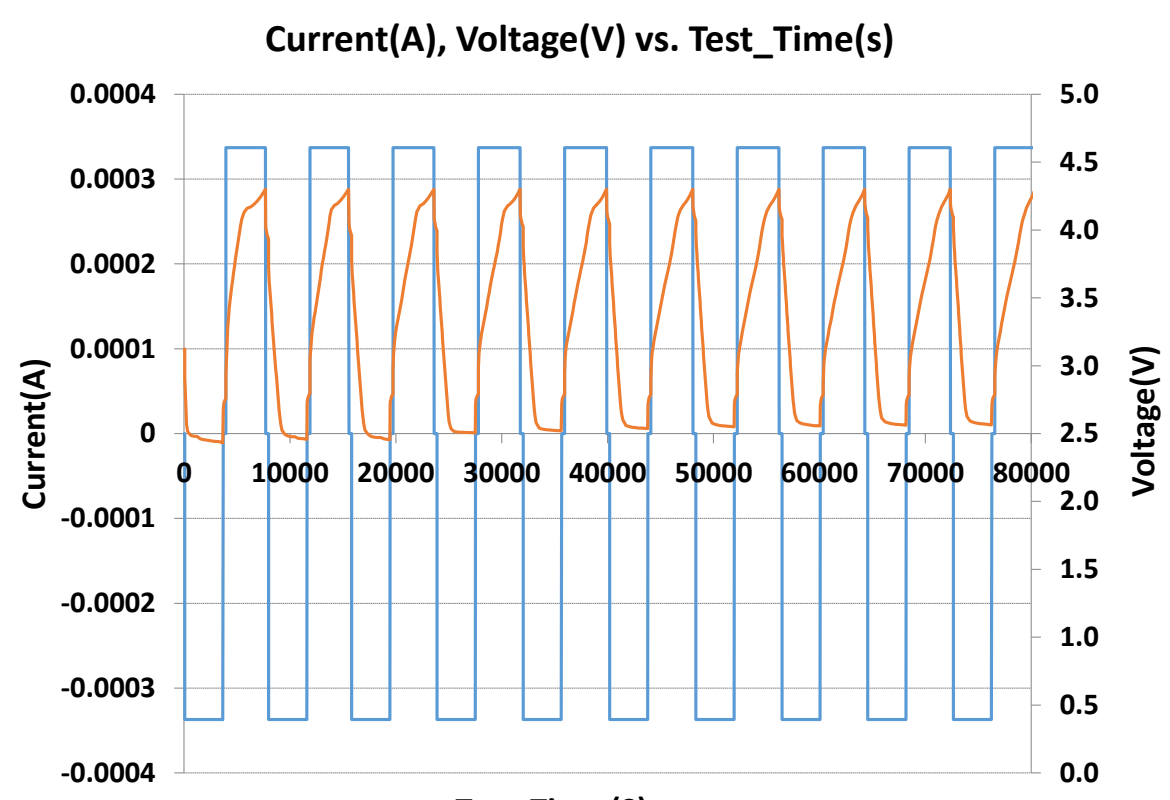

Test_Time (S)

Capacity vs. Cycle \#

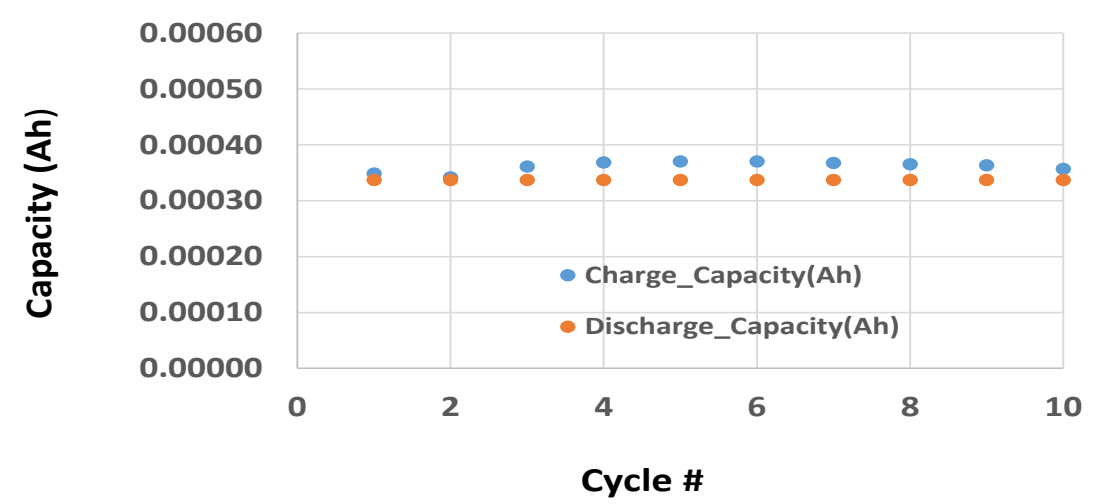

\section{$150 \mathrm{~mA} / \mathrm{g}$, After 300, 450, $300 \mathrm{~mA} / \mathrm{g}$}

Current(A), Voltage(V) vs. Test_Time(s)

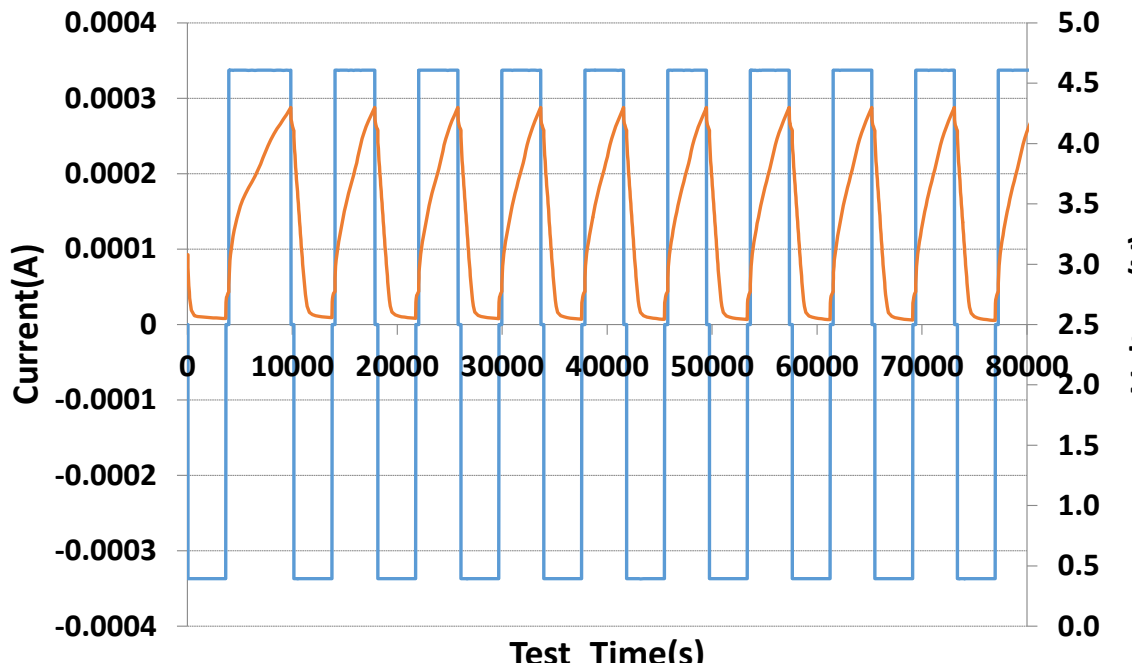

Capacity vs. Cycle \#

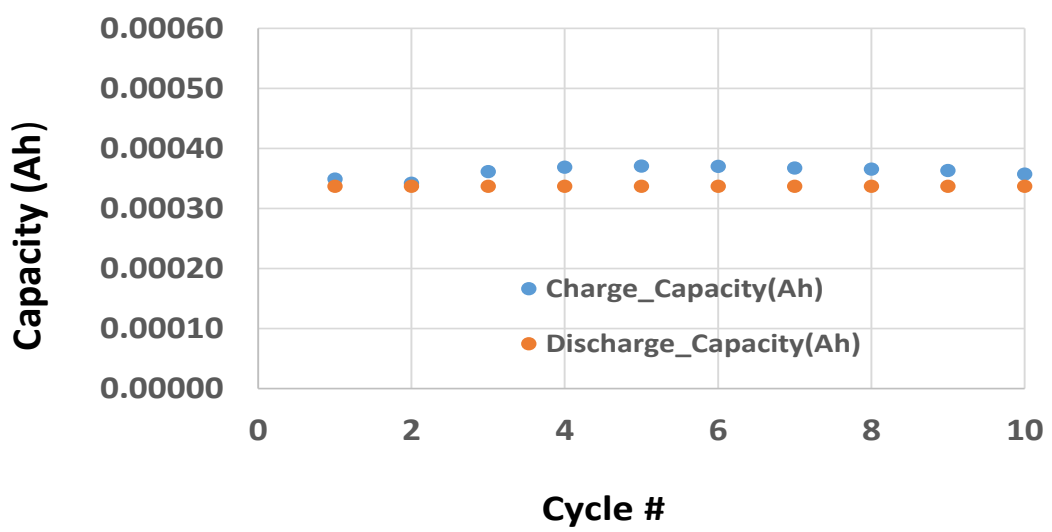




\section{Nagh 300 mA/g: Before and After Rate Capability Cycling}
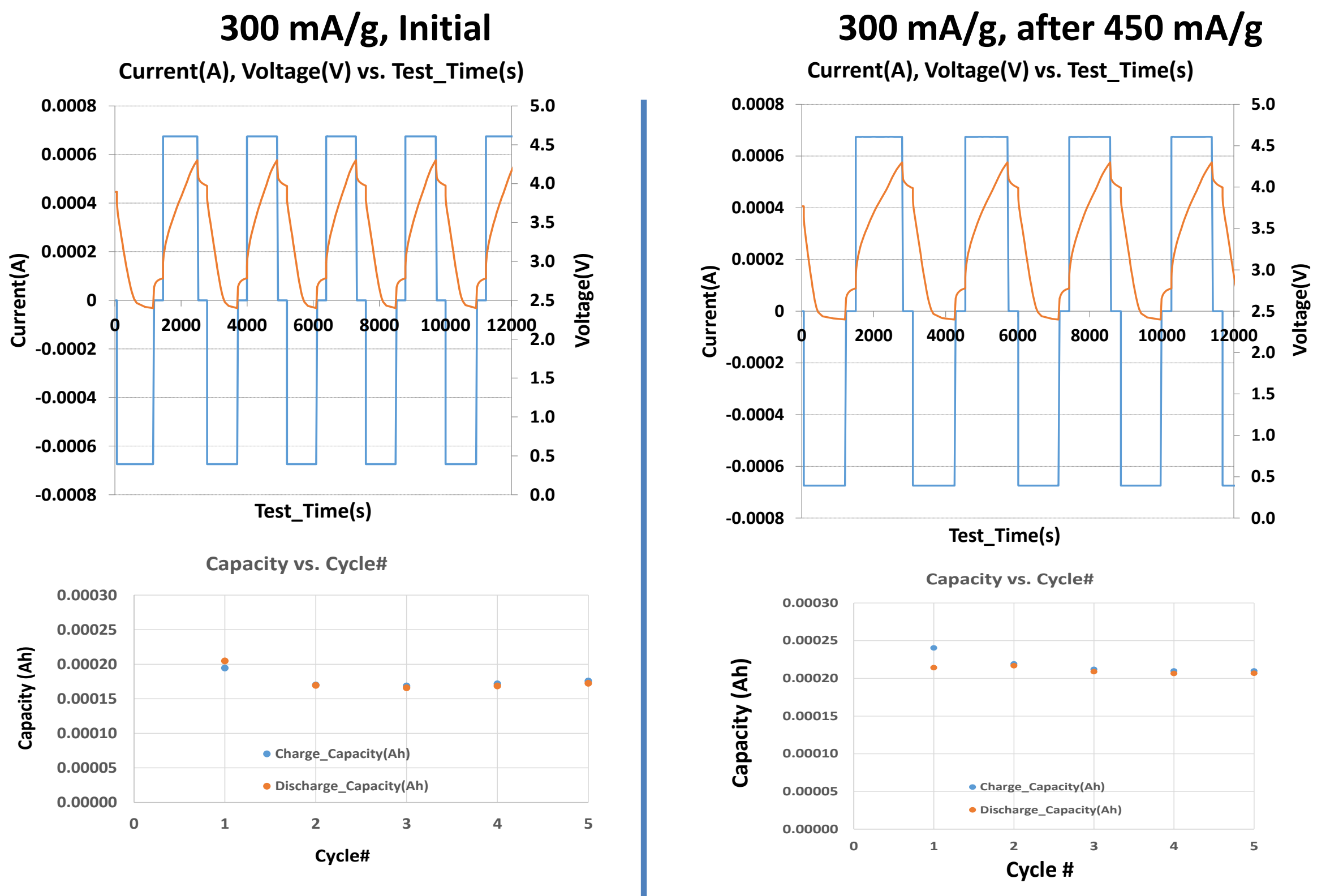


\section{Rate Capability Cycling vs. Commercial Air Cathode}

\begin{tabular}{|c|c|c|c|c|c|}
\hline \multicolumn{6}{|c|}{ Ketjen Carbon Black on Ni foam } \\
\hline Loading & $\mathrm{g} / \mathrm{cm} 2$ & \multirow{2}{*}{ Discharge Condition } & Discharge Time & $\mathrm{W} / \mathrm{kg}$ & Wh/kg \\
\hline \multicolumn{2}{|c|}{ Rate } & & (minutes) & vs. electrode & vs. electrode \\
\hline $\mathrm{mA} / \mathrm{g}$ & $\mathrm{mA} / \mathrm{cm} 2$ & \multirow{4}{*}{1 hour or $2.4 \mathrm{~V}$} & & & \\
\hline 150 & 0.47 & & 60 & 401 & 401 \\
\hline 300 & 0.95 & & 18.2 & 815 & 247 \\
\hline 450 & 1.42 & & 5.2 & 1317 & 114 \\
\hline \multicolumn{6}{|c|}{ Commercial Air Cathodes } \\
\hline \multirow{3}{*}{$\begin{array}{c}\text { CeTech } \\
(8 \mathrm{mg} / \mathrm{cm} 2)\end{array}$} & 0.5 & \multirow{3}{*}{1 hour or $2.4 \mathrm{~V}$} & 12 & 45 & 9 \\
\hline & 1 & & 0.55 & 99 & 0.9 \\
\hline & 1.5 & & & & \\
\hline \multirow{3}{*}{$\begin{array}{c}\text { Elat } \\
(6 \mathrm{mg} / \mathrm{cm} 2)\end{array}$} & 0.5 & \multirow{3}{*}{1 hour or $2.4 \mathrm{~V}$} & 26 & 51 & 22 \\
\hline & 1 & & 1.8 & 106 & 3.2 \\
\hline & 1.5 & & & & \\
\hline
\end{tabular}




\section{Result Summary/Next Steps}

- The factors such as substrate type, carbon materials, porosity and discharge cut-off voltage impact air-cathode cycling performance

- Kenjet black carbon on 3D Ni foam substrate demonstrated excellent cycling performance and rate capability

- Current under Investigation/Next Steps:

- Catalyst

- Electrolyte with additive 


\section{Acknowledgements}

- Bill Bennett (NASA/GRC), Fred Dynys (NASA/GRC) Donald Dornbusch (NASA/GRC \& Case Western Reserve University)

- LiON project funded under NASA Convergent Aeronautics Solutions (CAS) Program 
Thank you!

Any Questions? 